\title{
Training of Advanced Cardiac Life Support Skills to Nursing Staff in Critical Areas of Care
}

\author{
Joseph Mpambara ${ }^{1}$, Jean Claude Musengimana ${ }^{1}$, Vianney Ruhumuliza ${ }^{1}$, Katie Carlson ${ }^{1}$ \\ ${ }^{1}$ King Faisal Hospital, Rwanda
}

\section{Background}

This advanced cardiac life support skills (ACLS) program was free of charge and the program was coordinated in collaboration with resuscitation service, unit managers and clinical mentors. Prior to ACLS certification, classes of Basic Life Support (BLS) were held every week for the staff nurses at King Faisal Hospital.

\section{Objectives}

The purpose of this innovation was threefold, to: 1.Improve outcomes of patients requiring resuscitation through the education of nurses; 2. Meet the hospital accreditation standards in critical areas. 3. Build a sustainable program by educating instructors about local BLS and Advanced Cardiac Life Support (ACLS) in King Faisal Hospital.

\section{Description}

This ACLS program was free of charge and coordinated in collaboration with unit managers and clinical mentors. Prior to ACLS certification classes, the BLS classes were held every week for the staff nurses at King Faisal Hospital. The BLS instructors were American Heart Association certified (AHA) instructors; clinical mentors from the Human Resources for Health $(\mathrm{HRH})$ program and hospital staff members working together using the standards of the American Heart Association certifying 200 BLS nurses. Nurses from critical areas such as intensive care, emergency department and the operating theatre were chosen to complete the ACLS certification, consequently 80 nurses became ACLS certified and 6 nurses completed ACLS instructors training.

\section{Results}

Statistics from March 2014 indicated the survival rate following resuscitation efforts was 34\% and by October 2014 the survival rate had risen to $52 \%$.

\section{Lessons Learned}

This educational innovation represents a very positive collaboration between stakeholders in addressing the need for capacity building related to cardiac life support in Rwanda. Ideally there needs to exist continued educational opportunities with ACLS recertification and continued resuscitation review to maintain the high quality standards initiated with this program.

\section{Conclusions}

This intervention showed an obvious positive impact on health care delivery system at King Faisal Hospital, with a significant increase in the number of survivors due to earlier intervention and the skills learned at the ACLS program. All three of the preliminary purposes of the program were met. Our future goals include becoming the educational Centre for Rwanda in Advanced Cardiac Life Support.

Keywords: advanced cardiac life support, basic life support, human resources for health, American heart association 